\title{
Sensorimotor Memory For Fingertip Forces: Evidence For A Task-Independent Motor Memory
}

\author{
Barbara M. Quaney, Diane L. Rotella, Clayton Peterson, and Kelly J. Cole \\ Department of Exercise Science, The University of Iowa, Iowa City, Iowa 52241
}

When repetitively lifting an object with randomly varying mechanical properties, the fingertip forces reflect the previous lift. We examined the specificity of this "sensorimotor memory" by observing the effects of an isolated pinch on the subsequent lift of a known object. In this case, the pinch force was unrelated to the fingertip forces necessary to grip the object efficiently. The peak grip force used to lift the test object ( $4 \mathrm{~N}$ weight) depended on the preceding task. Compared with repetitively lifting the $4 \mathrm{~N}$ test object, the peak grip force was $2 \mathrm{~N}$ greater when a lift of the same object was preceded by a lift in which a hidden mass was attached to the object to increase the weight to 8 $\mathrm{N}$. This $2 \mathrm{~N}$ increase in grip force also occurred when subjects lifted the $4 \mathrm{~N}$ test object after pinching a force transducer with a force of 8 $\mathrm{N}$. Thus, similar grip forces were stored in sensorimotor memory for both tasks, and reflected subjects' use of $7.9 \pm 1.1 \mathrm{~N}$ to lift the $8 \mathrm{~N}$ object. Similar effects occurred when the preceding pinch or lift was performed with the opposite hand. The peak lift force was unaffected by the isolated pinch, suggesting that a generalized increase in fingertip and limb forces did not occur. We conclude that the sensorimotor memory is not specific for lifting an object. It is doubtful that this particular memory stores the physical properties of objects or reflects a forward internal model for predictively controlling fingertip forces.

Key words: grip force; memory; prediction; internal model; grasping; feedforward control

\section{Introduction}

We scale our fingertip forces predictively to handle objects with a secure, economical grip (Johansson, 1996). Several processes are at work. Visual analysis of the object size and shape appear to produce well scaled forces through visuomotor transformations, which may not involve memory except perhaps for information about density (Gordon et al., 1991b; Jenmalm and Johansson, 1997; Jenmalm et al., 2000). We also use associative processes to link the identity of an object with the necessary fingertip forces (Gordon et al., 1993; Dubrowski et al., 1999; Cole and Rotella, 2002). This memory representation is learned within a few lifts of the object and maintained in long-term memory, as reflected in our capacity to program the forces accurately $24 \mathrm{hr}$ later (Gordon et al., 1993).

There is another memory process that influences fingertip forces when objects are lifted repetitively. Random changes in weight or surface texture cause us to scale our fingertip forces according to the previous lift. Johansson and coworkers (Johansson and Westling, 1984, 1988; Jenmalm and Johansson, 1997) referred to this phenomenon as a "sensorimotor memory". This memory competes with visuomotor and associative memory mechanisms for scaling fingertip forces, indicating that it is a separate process (Gordon et al., 1991a; Salimi et al., 2000b; Cole and Rotella, 2002). This sensorimotor memory was manifest as a force "error" of 10-15\% compared with the force the subject would use during repetitive lifts of an object with constant mechanical properties (Cole and Rotella, 2002). This memory effect

\footnotetext{
Received Aug. 16, 2002; revised Nov. 25, 2002; accepted Dec. 18, 2002.

This work was supported by National Institutes of Health-National Institute on Aging Grant R01 AG12557. We thank Dr. Randolph Nudo for his helpful comments on this manuscript.

Correspondence should be addressed to Dr. Barbara Quaney, University of Kansas Medical Center 170A Landon Center on Aging, 3599 Rainbow Boulevard, Kansas City, KS 66160. E-mail: bquaney@kumc.edu.

Copyright $\odot 2003$ Society for Neuroscience $\quad 0270-6474 / 03 / 231981-06 \$ 15.00 / 0$
}

also is observed when the previous lift is performed with the opposite hand (Gordon et al., 1994).

Understanding the memory processes underlying anticipatory programming for fingertip forces enhances our knowledge of successful grasping during object manipulation. Therefore, we sought to determine whether this sensorimotor memory is restricted to the task of repetitively lifting an object, in part to address whether the content of this memory reflects the physical properties of the object, such as the weight. Here, we studied the effects of an isolated pinch on the fingertip forces subsequently used to lift a known object, either with the same or the opposite hand. Subjects intermittently pinched (but did not lift) a standard pinch force transducer before lifting the test object. Neither the pinch forces exerted against the transducer nor the physical properties of the transducer were relevant to the task of specifying the fingertip forces for the subsequent lift of the familiar object. Findings of a strong effect from the preceding pinch when lifting a familiar object would be inconsistent with a task-specific memory or a memory that stores the mechanical properties of objects.

\section{Materials and Methods}

Subjects. Twenty-one young, healthy, right-hand-dominant adults voluntarily participated in three experiments. Ten subjects (three males, seven females; $19-32$ years of age) participated in the first two experiments, in which we studied the dominant hand. Eleven additional subjects (five males, six females; 20 -34 years of age) volunteered for the final experiment, in which the contralateral hand was also studied. Subjects had no history of previous upper-limb injury and possessed normal grip strength and range of motion. They were unaware of the purpose of these experiments and had not previously participated in experiments within this laboratory. Informed consent was obtained from subjects according to the Declaration of Helsinki. The University of Iowa Human Subjects Internal Review Board approved the experiments.

Apparatus. The novel object that subjects lifted (test object) has been described previously (Forssberg et al., 1991) (Fig. 1). It had two parallel 


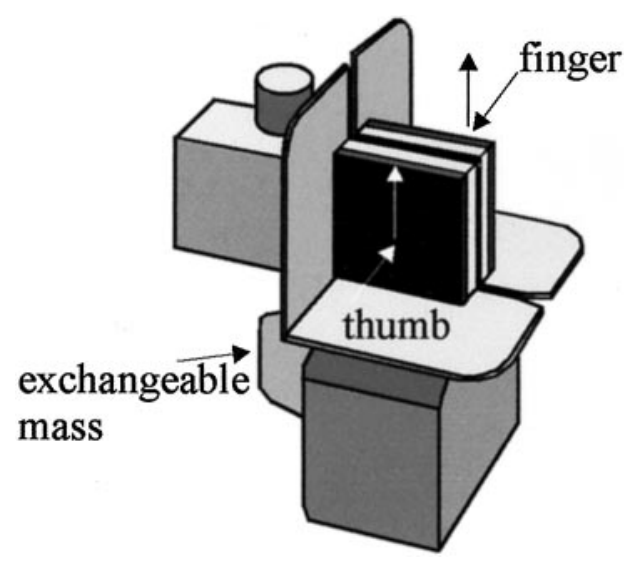

Figure 1. Novel test object. Subjects grasped and lifted the test object at the black, sandpaper-covered vertical surfaces using the thumb and index finger.

Plexiglas grip surfaces $(35 \times 35 \mathrm{~mm})$ that were oriented vertically and separated by a distance of $2.2 \mathrm{~cm}$. The grip surfaces were covered with black \#320 grit sandpaper. Load cells embedded in the object measured the force normal to the grip surfaces (grip force), and the vertical tangential force (lift force) separately for the thumb and index finger. The linearity, repeatability, and hysteresis of the test object were computed to be $\pm 0.8 \%, \pm 1.86 \%$, and $\pm 1.1 \%$, respectively, of full scale. An accelerometer (SenSyn Model SXL010G; Sunnyvale, CA) affixed to the object measured vertical acceleration. Rigid shrouds in the vertical and horizontal planes of the object prevented the subject's fingertips from hooking around the edges of the grip surfaces. Inserting various weights into a cavity within the object changed the total weight of the test object.

A mechanical pinch force transducer with an analog dial gauge (model PG-60; B\&L Engineering, Santa Fe Springs, CA) was used for the task involving pinching to various force levels. The range of available force measurements was from 0 to $28 \mathrm{~kg}$ (accuracy, $\pm 1 \%$ ). The weight, shape, and frictional surfaces of the pinch transducer were completely different from the mechanical properties of the test object (see above). During the experiment, subjects merely pinched the transducer without lifting it.

Procedure. Subjects washed their hands with soap and water and then sat in front of the testing table. The test object was positioned on the adjustable table so that reaching for the object required only minimal shoulder flexion without internal rotation, external rotation, or abduction. During the lifting trials, subjects grasped the test object using a precision grip with the thumb and index finger, with the wrist in slight extension. The test object was lifted using primarily elbow flexion while

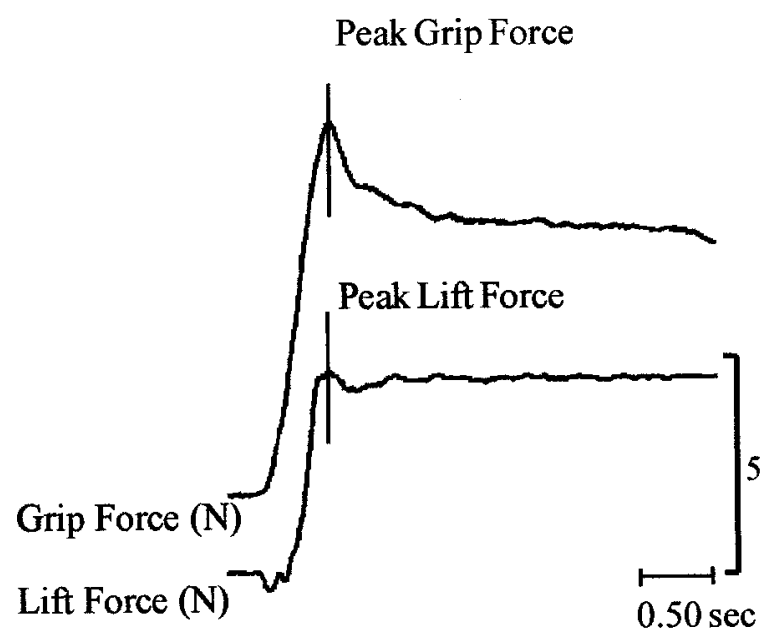

Figure 2. Single representative trials of a typical subject lifting the $4 \mathrm{~N}$ test object. Peak grip and lift forces (vertical lines) occur after the object has been lifted from the support surface.
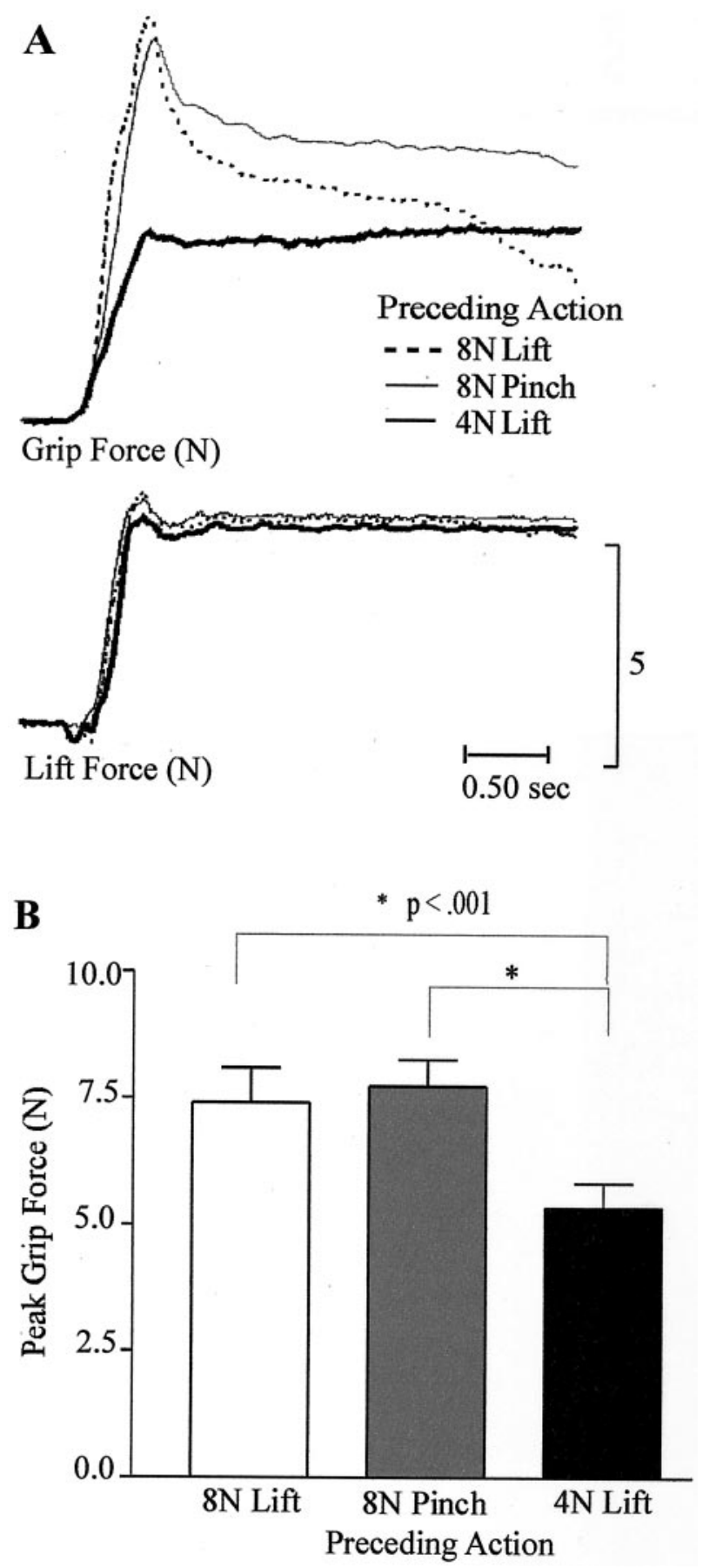

Figure 3. The peak grip force when lifting the $4 \mathrm{~N}$ test object depends on the preceding action. $A$, Fingertip force records of single trials from a typical subject for lifts of the $4 \mathrm{~N}$ test object when preceded by an $8 \mathrm{~N}$ pinch (thin line), a lift of the $8 \mathrm{~N}$ test object (dashed line), or another $4 \mathrm{~N} \mathrm{lift}$ of the test object (thick line). B, Average peak grip forces for the group across conditions (lines indicate SEM), when a lift of the $4 \mathrm{~N}$ object was preceded by an $8 \mathrm{~N} \mathrm{lift}$ (white), an $8 \mathrm{~N}$ pinch ( gray), or a $4 \mathrm{~N} \mathrm{lift} \mathrm{(black).} \mathrm{Average} \mathrm{peak} \mathrm{grip} \mathrm{forces} \mathrm{were} \mathrm{significantly} \mathrm{greater} \mathrm{for}$ lifts of the $4 \mathrm{~N}$ test object when preceded by $8 \mathrm{~N}$ pinches and $8 \mathrm{~N}$ lifts compared with repeated 4 $\mathrm{N}$ lifts. ${ }^{*} p<0.001$.

maintaining a neutral forearm position (neither supinated nor pronated). Subjects lifted the test object $\sim 5-8 \mathrm{~cm}$ from the table, held it stationary in the air for $\sim 5 \mathrm{sec}$, and then lowered the object back to the table.

Each subject rested their right forearm on the testing table to perform the pinches. The experimenter held the pinch transducer while subjects used their right thumb and index finger to squeeze the distal tips of the transducer to the desired force level and maintained this force for $7 \mathrm{sec}$. 


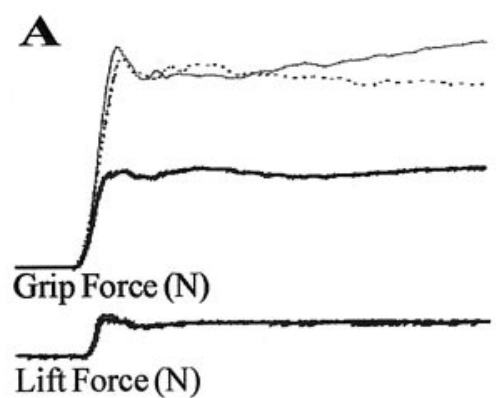

PRECEDING ACTION

... $8 \mathrm{~N}$ Lift, same hand

- $8 \mathrm{~N}$ Lift, opposite hand

- $2 \mathrm{~N}$ Lift, same hand

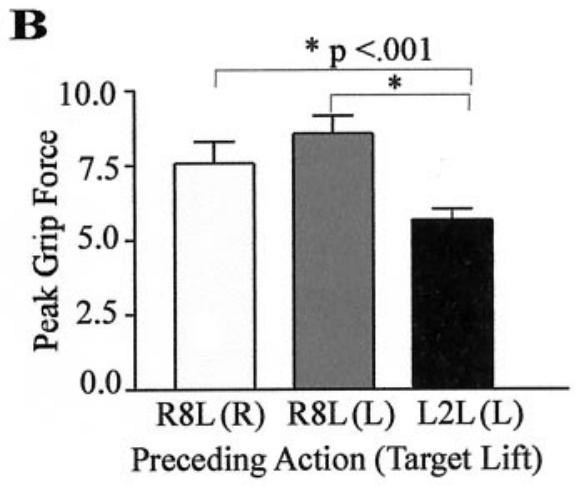

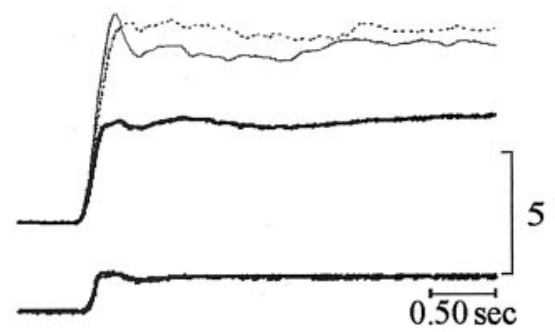

PRECEDING ACTION

... 8N Pinch, same hand

- 8N Pinch, opposite hand

- $2 \mathrm{~N}$ Lift, same hand

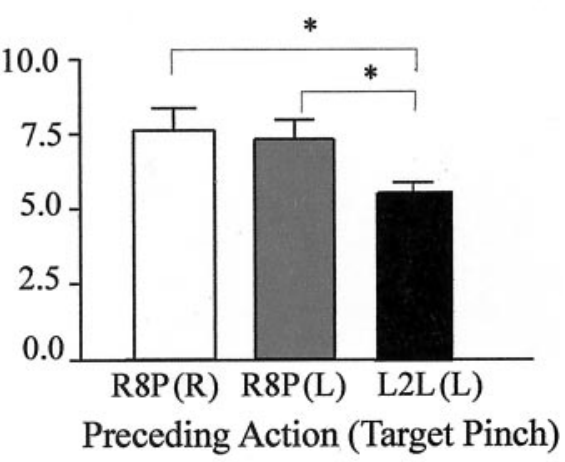

index finger in the manner described in the first experiment. Each subject performed a total of 45 lifts of the $4 \mathrm{~N}$ test object. We analyzed the fingertip forces for seven lifts of the $4 \mathrm{~N}$ test object when immediately preceded by either an $8 \mathrm{~N}$ pinch or another $4 \mathrm{~N}$ lift.

In the final experiment, we tested whether a pinch to $8 \mathrm{~N}$ with their right (dominant) thumb and index finger influenced the fingertip forces when subjects lifted the $2 \mathrm{~N}$ test object with their left (nondominant) thumb and index finger. The following tasks preceded the left-handed lifts of the $2 \mathrm{~N}$ test object: (1) righthanded lifts of the test object $(2$ or $8 \mathrm{~N})$ in a pseudorandom order and (2) right-handed pinches of the pinch force transducer to either 2 or $8 \mathrm{~N}$. A total of 128 trials were performed in this experiment. Seven trials of each of the following types were analyzed: (1) left-handed lift of the $2 \mathrm{~N}$ test object when preceded by the right-handed lift of the $8 \mathrm{~N}$ object, (2) lefthanded lift of the $2 \mathrm{~N}$ test object when preceded by the right-handed $8 \mathrm{~N}$ pinch, (3) left-handed lift of the $2 \mathrm{~N}$ test object when preceded by another lift of the $2 \mathrm{~N}$ object with the left hand, and (4) right-handed lift of the $2 \mathrm{~N}$ test object when preceded by either an $8 \mathrm{~N}$ lift or an $8 \mathrm{~N}$ pinch with the right hand.

Data collection and analysis. Force and accelerometer signals were collected and analyzed using a personal computer with DataPac 2000 software (Run Technologies, Laguna Hills, CA). All signals were sampled with an analog-to-digital converter at 16 bit resolution. The grip force, load force, and acceleration of the test object were sampled at a rate of 400 samples/sec. Grip force was calculated as the mean of the normal force measured at the index finger and thumb (normal finger + nor$\left.\mathrm{mal}_{\text {thumb }}\right) / 2$. The vertical load force signals were summed at both digits to provide the lift force (tangential finger + tangential $\left._{\text {thumb }}\right)$. The accelerometer signal was used to determine the onset of vertical motion to detect when the test object had lifted from the table, as well as peak acceleration. The peak grip and lift forces were measured after

Therefore, subjects performed all pinches without lifting the pinch transducer. Subjects performed these right-handed pinches with the wrist slightly extended and the forearm in a supinated position.

The first experiment examined the fingertip forces applied while lifting a $4 \mathrm{~N}$ object when immediately preceded by a lift of either the 4 or $8 \mathrm{~N}$ object. Subjects lifted the object with the right thumb and index finger using a precision grip. Between each lift, the test object weight was changed to 2,4 , or $8 \mathrm{~N}$ in a pseudorandom order. Subjects were unaware of the weight of the test object before lifting it from the table. Repeated lifts of the test object when the weight was $4 \mathrm{~N}$ were embedded within this pseudorandom order. Each subject performed a total of 47 lifts of the test object with these weights. Seven lifts of each of the following were analyzed in the statistical model: (1) lifts of the test object at the $4 \mathrm{~N}$ weight when immediately preceded by a lift of the $8 \mathrm{~N}$ weight, and (2) lifts of the test object at the $4 \mathrm{~N}$ weight when preceded by another $4 \mathrm{~N}$ weight.

In the second experiment, we examined the fingertip forces exerted when lifting the $4 \mathrm{~N}$ test object after performing the $8 \mathrm{~N}$ pinch task. The weight of the test object remained constant at $4 \mathrm{~N}$ throughout this experiment. After the first lift in this experiment, subjects then had prior knowledge of the object weight for each of the subsequent lifts and were aware that the mass within the hidden cavity of the object was not changed. Using their right thumb and index finger, each subject randomly pinched the pinch force transducer to a sustained force of 2, 4, or $8 \mathrm{~N}$ between lifts of the $4 \mathrm{~N}$ test object. Within $10 \mathrm{sec}$ after each pinch, subjects grasped and lifted the $4 \mathrm{~N}$ test object with their right thumb and lift-off of the object for each trial (Fig. 2).

The order of the pinch and lift experiments was counterbalanced across subjects. The trials were averaged within each subject and compared between conditions using repeated-measures ANOVA for the factors group $\times$ preceding action (pinch $8 \mathrm{~N}$, lift $8 \mathrm{~N}$, lift $4 \mathrm{~N}$, lift $2 \mathrm{~N}$ ). Tukey's honest significant difference tests were used for post hoc analyses. Values reported are group means \pm SEM.

\section{Results}

Lifting the object when the weight was $8 \mathrm{~N}$ yielded a peak grip force $(7.9 \pm 1.1 \mathrm{~N})$ that was comparable with the $8 \mathrm{~N}$ pinch target force. This result confirms that the target force on the pinch trials was appropriate for comparing the facilitative effects of pinching versus lifting the $8 \mathrm{~N}$ object.

The peak grip force used to lift the $4 \mathrm{~N}$ test object with the right hand depended on the weight of the object on the preceding lift, which replicates the often-reported sensorimotor memory (Fig. 3 ). An isolated pinch to $8 \mathrm{~N}$ yielded a peak grip force on the subsequent lift of the $4 \mathrm{~N}$ object that also was substantially greater $(7.6 \pm 0.5 \mathrm{~N})$ than the force used on repeated lifts of the $4 \mathrm{~N}$ object $(5.3 \pm 0.5 \mathrm{~N})$. The size of the effect from a preceding pinch was comparable with that observed when a lift of the $4 \mathrm{~N}$ object was immediately preceded by a lift of the $8 \mathrm{~N}$ object $(7.4 \pm 0.8 \mathrm{~N})$. 
To compare these results statistically, we subtracted the peak grip force for a lift of the $4 \mathrm{~N}$ test object when preceded by another $4 \mathrm{~N}$ lift from the peak grip force for a lift of the $4 \mathrm{~N}$ test object when preceded by an $8 \mathrm{~N}$ pinch $(2.3 \pm 0.5 \mathrm{~N})$. We also calculated the difference between the lifts of the $4 \mathrm{~N}$ test object when preceded by an $8 \mathrm{~N}$ lift and repeated lifts of the $4 \mathrm{~N}$ object $(2.1 \pm 0.5 \mathrm{~N})$. These values were not different $\left(F_{(2,8)}=0.09 ; p=0.77\right)$. All 10 subjects studied in the first two experiments programmed higher peak grip forces when the $8 \mathrm{~N}$ pinch preceded a lift of the $4 \mathrm{~N}$ object compared with repeatedly lifting the $4 \mathrm{~N}$ object.

The increased grip force during lifts of the $4 \mathrm{~N}$ object when preceded by either an $8 \mathrm{~N}$ pinch or an $8 \mathrm{~N}$ lift persisted into the static phase of the task, when the object was held stationary above the table (Fig. 3A). A few seconds after the peak grip force was achieved, the grip force averaged $5.8 \mathrm{~N}$ after an $8 \mathrm{~N}$ pinch and 5.7 $\mathrm{N}$ after a lift of the $8 \mathrm{~N}$ object ( $p>0.86$ ). These values were less than the peak grip forces described previously $(\sim 7.5 \mathrm{~N})$ but were greater than the static phase grip force when the $4 \mathrm{~N}$ object was lifted repeatedly $(4.8 \mathrm{~N} ; p<0.01)$. Therefore, a complete adjustment to the altered object weight requires more than one lift of the light object (Johansson and Westling, 1988); likewise, more than one lift of the light object after a pinch to $8 \mathrm{~N}$ is required to eliminate the effects of the pinch. This suggests that similar mechanisms are at work for the sensorimotor memory and the effects after a pinch.

Pinching or lifting with the right hand affected the peak grip forces used on the subsequent lift of the $2 \mathrm{~N}$ object with the left hand (Fig. 4). The peak grip forces for a left-handed lift of the $2 \mathrm{~N}$ test object when preceded by an $8 \mathrm{~N}$ lift with the right hand $(8.6 \pm$ $0.7 \mathrm{~N})$ or an $8 \mathrm{~N}$ pinch with the right hand $(7.4 \pm 0.6 \mathrm{~N})$ were significantly greater compared with a lift of the $2 \mathrm{~N}$ test object when preceded by another $2 \mathrm{~N}$ lift $(5.7 \pm 0.4 \mathrm{~N})\left(F_{(20,20)}=14.7\right.$; $p=0.001)$. The size of the effect on the peak grip force for the left-handed $2 \mathrm{~N}$ lift of the object when preceded by a righthanded $8 \mathrm{~N}$ lift $(2.9 \pm 0.6 \mathrm{~N})$ was greater than the $2 \mathrm{~N}$ lift of the object with the left hand when preceded by a right-handed $8 \mathrm{~N}$ pinch $(1.7 \pm 0.3 \mathrm{~N})$ and was nearly significant $\left(F_{(1,10)}=4.83 ; p=\right.$ 0.053).

The effects of the isolated pinch on the next lift of the $4 \mathrm{~N}$ test object with the same hand were not simply generalized increases in force at the fingertips. The isolated pinch affected only the grip force and not the peak lift force used to lift the $4 \mathrm{~N}$ object vertically (Fig. 5). When comparing the three conditions with repeated-measures ANOVA, there was a significant main effect for the peak lift forces, as expected when lifting identicalappearing objects of different weights in random order (Johansson and Westling, 1988). The simple effects for the peak lift force when a right-handed $8 \mathrm{~N}$ pinch preceded a right-handed lift of the $4 \mathrm{~N}$ test object $(4.8 \pm 0.1 \mathrm{~N})$ were not different from lifts of the $4 \mathrm{~N}$ test object when preceded by another $4 \mathrm{~N}$ lift $(4.7 \pm 0.1 \mathrm{~N}$; $p=0.24$ ) with the right hand. However, both of these peak lift forces were significantly different from the peak lift force when the $8 \mathrm{~N}$ lift preceded the $4 \mathrm{~N}$ lift $\left(6.1 \pm 0.17 ; F_{(2,8)}=10.89 ; p<\right.$ $0.001)$. The peak lift force rates were similar between lifts of the 4 $\mathrm{N}$ object when preceded by an $8 \mathrm{~N}$ pinch $(37.6 \pm 13.7 \mathrm{~N} / \mathrm{sec})$ and a $4 \mathrm{~N}$ lift $(36.1 \pm 16.6 \mathrm{~N} / \mathrm{sec})$, whereas peak lift force rates were greater when preceded by an $8 \mathrm{~N}$ lift $(41.2 \pm 17.8 \mathrm{~N} / \mathrm{sec})$. Likewise, the effect of a pinch or an object lift with the hand opposite to the subsequent lift was restricted to the grip force. There was no difference in the peak lift force when a left-hand lift of the $2 \mathrm{~N}$ test object was preceded by another left-hand lift of the $2 \mathrm{~N}$ test object $(2.7 \pm 0.1 \mathrm{~N})$ or an $8 \mathrm{~N}$ pinch with the right hand $(2.8 \pm 0.1$ N) $\left(F_{(2,20)}=1.99 ; p=0.16\right)$.

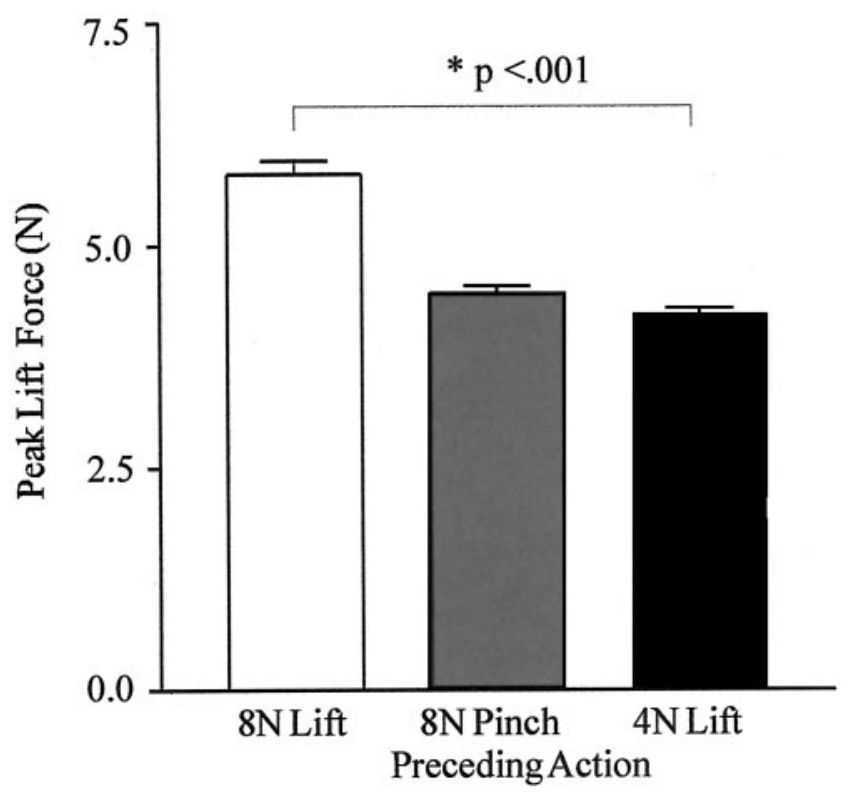

Figure 5. Peak lift force when a lift of the $4 \mathrm{~N}$ test object was preceded by an $8 \mathrm{~N}$ lift (white), $8 \mathrm{~N}$ pinch ( gray), or another $4 \mathrm{~N} \mathrm{lift}$ (black). An $8 \mathrm{~N}$ pinch preceding a $4 \mathrm{~N}$ lift had no effect on the peak lift force. However, an $8 \mathrm{~N}$ lift preceding a $4 \mathrm{~N}$ lift produced significantly greater peak lift forces $\left({ }^{*} p<0.001\right)$ compared with repeated $4 \mathrm{~N}$ lifts.

Finally, the effect of pinching was robust across individual trials for each subject. There was a relatively small difference in the peak grip force when lifting the object with the right hand after the first $8 \mathrm{~N}$ pinch $(8.9 \pm 2.9 \mathrm{~N})$ compared with the last $8 \mathrm{~N}$ pinch $(7.6 \pm 1.8 \mathrm{~N})\left(t_{(1,9)}=1.9 ; p<0.09\right)$. This same effect was also observed when lifting the object with the opposite hand used to perform the pinches. The peak grip force for the $2 \mathrm{~N}$ lift after the first $8 \mathrm{~N}$ pinch trial $(7.7 \pm 2.7 \mathrm{~N})$ was similar to the lift after the last $8 \mathrm{~N}$ pinch $(7.6 \pm 2.1 \mathrm{~N})\left(t_{(1,10)}=0.1 ; p<0.97\right)$.

\section{Discussion}

A preceding action significantly affects the peak grip force when handling objects. This well known phenomenon occurs when subjects lift an object that randomly varies in mechanical properties from lift to lift. This same effect on the peak grip force is observed when the subsequent lift is performed with the hand ipsilateral or contralateral to the preceding target lift (Johansson and Westling, 1988; Forssberg et al., 1992; Gordon et al., 1994; Salimi et al., 2000b). Fingertip forces are programmed according to the requirements of the preceding lift, particularly when visual cues are not available during sequential lifts of an object (Johansson and Westling, 1988; Jenmalm and Johansson, 1997). This same result occurs when significant visual geometric cues are unavailable to indicate the mechanical properties of the object, such as the center of mass (Salimi et al., 2000a,b). Witney et al. (2001) extended these fundamental observations using an apparatus that mimicked pulling an object with one hand while restraining the object with the other hand. They demonstrated that the fingertip forces depended strongly on the most recent preceding trial and more weakly on the second and third preceding trials.

Here, we demonstrate a similar effect when subjects simply pinched an unrelated object before lifting the familiar test object with known mechanical properties. Neither the target pinch forces nor the mechanical properties of the pinch force transducer were relevant to programming the fingertip forces for the 
subsequent lift of the test object. The effect of the pinches on the peak grip force for the subsequent lift of the lightweight test object did not diminish over the course of the experiments. Furthermore, a strong pinch influenced only the grip forces and not the lift forces when subsequently lifting the test object. This finding demonstrates that the pinch did not simply facilitate upper-limb forces in a nonspecific manner. These effects were observed regardless of the hand performing the preceding pinch.

The memory used to predict the motor commands needed to handle objects has been interpreted to reflect forward and inverse internal models (Flanagan and Wing, 1993, 1995; Kawato, 1999; Witney et al., 2001). When viewed simply, the results from the present experiment are inconsistent with an internal model that uses the mechanical properties of an object for predicting fingertip forces. These models allow us to predict the consequences of our motor commands by causally relating the motor commands to the actual outcomes using efferent copy (Massion, 1992; Wolpert et al., 1995; Blakemore et al., 1998). Forward models require representations of both our body and the external world. When viewed in this way, the sensorimotor memory studied here appears to represent the most recent motor action, rather than directly representing the external world, such as the mechanical properties of the test object (Flanagan and Wing, 1993). However, other memory representations used to set fingertip forces predictively, as discussed below, may reflect internal models that operate using object properties.

Our experiment demonstrated the effect of a pinch on the grip component for the subsequent lift of a familiar object. Generalizing our results to memory mechanisms that predictively scale other forces during a grip and lift task must be done with caution. Rather than a single memory representation of the fingertip forces for lifting objects that participates in an internal model, our results may fit more complex views of predictive force generation. One alternative explanation may be that different force scaling mechanisms are used for the gripping and lifting components of the task. Perhaps we use an object-based memory when lifting an object but an action-based memory when pinching, given that object properties such as load are unavailable or irrelevant. It is also possible that our observation of the grip force information transferring between the unrelated tasks reflects multiple mechanisms for scaling fingertip forces. There may be a more general mechanism for scaling grip forces that uses a simple mechanism for scaling in relation to parameters such as friction at the gripped surfaces. Furthermore, although our experimental results cannot directly address this issue, scaling for the object load may be under more direct control of mechanisms based on internal models.

The utility of a memory of the previous motor action may be useful in learning motor actions in daily life given that truly unpredictable variations in the external world are seldom encountered. A similar phenomenon was reported recently for reaching with the arm against a viscous force field that varied in amplitude unpredictably across trials (Scheidt et al., 2001). Despite the random variation in perturbation amplitude across trials, subjects produced arm movements that reflected the preceding trial. Thus, when external conditions (force fields, object properties) vary randomly, our upper-limb forces reflect the previous motor action. In more predictable environments, a similar one-trial sensorimotor effect on the fingertip forces used to handle objects is substantially blunted. This is observed after subjects learn to identify and associate other cues with the external conditions for successfully predicting the needed motor commands, such as visually identifying a familiar object (Gordon et al., 1991a; Cole and
Rotella, 2002), when geometric cues about object size are available (Gordon et al., 1991a), or when sensory information is incompatible with the ongoing action (Blakemore et al., 1998). As noted previously, this persistence of the sensorimotor memory effect, although reduced in size, suggests that this memory is independent of other associative mechanisms that help to predict and generate the required fingertip forces. Other recent work has also suggested that several memory representations may underlie our ability to perform the complex motor act of gripping and lifting an object (Edin et al., 1992; Burstedt et al., 1997; Salimi et al., 2000a,b).

There is evidence that the trial-to-trial sensorimotor memory decays rapidly with time, also supporting the view that this memory may be independent of other mechanisms for predictively scaling fingertip forces. According to a recent report, the sensorimotor memory for the friction at the gripped surfaces begins to decay within 10 sec of releasing the object, based on the drifting of the grip force on the subsequent trial. In contrast, the associative memory for friction based on object color persists $24 \mathrm{hr}$ later (Sinnaeve et al., 2002). Likewise, in a separate experiment, we first had subjects lift a novel object using self-selected grip forces. They returned $4 \mathrm{hr}$ later to once again repetitively lift the same object by generating a pinch grasp that far exceeded the weight of the object. During testing on the following day, subjects predictively generated grip forces that matched the weight of the object. Therefore, the sensorimotor memory acquired from a strong pinch apparently decays over $24 \mathrm{hr}$ and does not produce lasting interference effects (Quaney and Cole, 2002).

It is unclear whether the grip force effects attributable to an unrelated pinch action could be replicated by a more generalized exertion of muscular force before lifting the test object. Previous data from our laboratory demonstrate that fingertip forces are slightly increased when grasping an object with one hand while simultaneously performing rapid knee extensions (Werremeyer and Cole, 1997). It is not known whether this effect persists after a delay, such as performing a strong voluntary contraction of an unrelated muscle group before lifting a familiar object.

Our observation that the memory transfers across hands for both a preceding pinch and lift indicates the participation of the cerebral cortex, with information most likely transferred via the corpus callosum, based on reports from a patient with callosal agenesis (Gordon et al., 1994). The strong one-trial effect exceeds expectations for mediation through the primary motor cortex (Classen et al., 1998). The cerebellum has been suggested as a critical site for constructing and manipulating forward internal models based on functional imaging studies in humans (Imamizu et al., 2000; Ito, 2000).

We conclude that multiple memories underlie our ability to predictively generate fingertip forces to manipulate an object. The so-called sensorimotor memory may be based on the motor commands used to handle an object rather than on the mechanical properties of the object. It does not appear to be specific to handling objects, but may reflect a fundamental memory or forward model for learning actions in a stochastic environment.

\section{References}

Blakemore SJ, Goodbody SJ, Wolpert DM (1998) Predicting the consequences of our own actions: the role of sensorimotor context estimation. J Neurosci 18:7511-7518.

Burstedt MKO, Edin BB, Johansson RS (1997) Coordination of fingertip forces during human manipulation can emerge from independent neural networks controlling each engaged digit. Exp Brain Res 117:67-79. 
Classen J, Liepert J, Wise S, Hallett M, Cohen L (1998) Rapid plasticity of human cortical movement representation induced by practice. J Neurophysiol 79:1117-1123.

Cole K, Rotella D (2002) Old age impairs the use of arbitrary visual cues for predictive control of fingertip forces during grasp. Exp Brain Res 143:35-41.

Dubrowski A, Proteau L, Carnahan H (1999) Practice influences the coordination of visual and haptic inputs during grasping. Soc Neurosci Abstr 25:1913.

Edin BB, Westling G, Johansson RS (1992) Independent control of human finger-tip forces at individual digits during precision lifting. J Physiol (Lond) 450:547-564.

Flanagan JR, Wing AM (1993) Modulation of grip force with load force during point to point arm movements. Exp Brain Res 95:131-143.

Flanagan JR, Wing AM (1995) The stability of precision grip forces during cyclic arm movements with a hand-held load. Exp Brain Res 105:455-464.

Forssberg H, Eliasson A, Kinoshita H, Johansson R, Westling G (1991) Development of human precision grip. I. Basic coordination of force. Exp Brain Res 85:451-457.

Forssberg H, Kinoshita H, Eliasson A, Johansson R, Westling G, Gordon A (1992) Development of human precision grip. II. Anticipatory control of isometric forces targeted for object's weight. Exp Brain Res 90:393-398.

Gordon A, Forssberg H, Johansson R, Westling G (1991a) Visual size cues in the programming of manipulative forces during precision grip. Exp Brain Res 83:477-482.

Gordon A, Forssberg H, Johansson R, Westling G (1991b) Integration of sensory information during the programming of precision grip: comments on the contributions of size cues. Exp Brain Res 85:226-229.

Gordon A, Westling G, Cole K, Johansson R (1993) Memory representations underlying motor commands used during manipulation of common and novel objects. J Neurophysiol 69:1789-1796.

Gordon A, Forssberg H, Iwasaki N (1994) Formation and lateralization of internal representations underlying motor commands during precision grip. Neurophychologia 32:555-568.

Imamizu H, Miyauchi S, Tamada T, Sasaki Y, Takino R, Putz B, Yoshioka T, Kawato M (2000) Human cerebellar activity reflecting an acquired internal model of a new tool. Nature 403:192-195.
Ito M (2000) Internal model visualized. Nature 403:153-154.

Jenmalm P, Johansson R (1997) Visual and somatosensory information about object shape control manipulative fingertip forces. J Neurosci 17:4486-4499.

Jenmalm P, Dahlstedt S, Johansson R (2000) Visual and tactile information about object-curvature control fingertip forces and grasp kinematics in human dexterous manipulation. J Neurophysiol 84:2984-2997.

Johansson R (1996) Sensory control of dexterous manipulation in humans San Diego: Academic.

Johansson RS, Westling G (1984) Roles of glabrous skin receptors and sensorimotor memory in automatic control of precision grip when lifting rougher or more slippery objects. Exp Brain Res 56:550-564

Johansson R, Westling G (1988) Coordinated isometric muscle commands adequately and erroneously programmed for the weight during lifting task with precision grip. Exp Brain Res 71:59-71.

Kawato M (1999) Internal models for motor control and trajectory planning. Curr Opin Neurobiol 9:718-727.

Massion J (1992) Movement, posture and equilibrium: interaction and coordination. Prog Neurobiol 38:35-56.

Quaney B, Cole K (2002) Memory for grip force is not based upon the object properties. Soc Neurosci Abstr 28:665.4.

Salimi I, Frazier W, Gordon AM (2000a) Visual cues and digit specificity during precision grip. Soc Neurosci Abstr 26:831.3.

Salimi I, Hollender I, Frazier W, Gordon A (2000b) Specificity of interna representations underlying grasp. J Neurophysiol 84:2390-2397.

Scheidt RA, Dingwell JB, Mussa-Ivaldi FA (2001) Learning to move amid uncertainty. J Neurophysiol 86:971-985.

Sinnaeve A, Dubrowski A, Carnahan H (2002) Evidence for the use of both iconic and long-term memory systems for friction when grasping. Soc Neurosci Abstr 28:268.4.

Werremeyer MM, Cole K (1997) Wrist action affects precision grip force J Neurophysiol 78:271-280.

Witney AG, Vetter P, Wolpert DM (2001) The influence of previous experience on predictive motor control. NeuroReport 12:649-653.

Wolpert DM, Ghahramani Z, Jordon MI (1995) An internal model for sensorimotor integration. Science 269:1880-1882. 